\title{
Responses of water snakes (Natrix $r$. rhombifera) and garter snakes (Thamnophis sirtalis) to chemical cues*
}

\author{
RICHARD H. PORTER and JAMES A. CZAPLICKI \\ John F. Kennedy Center for Research on Education and Human Development \\ George Peabody College for Teachers, Nashville, Tennessee 37203
}

\begin{abstract}
In a series of simultaneous two-choice preference tests, water snakes (Natrix $r$. rhombifera) displayed a significant preference for a clean area of a test chamber vs an area soiled by a conspecific. No differential responsiveness was found for a clean area as compared to an area soiled by either a sympatric species of garter snake (Thamnophis sirtalis) or by the individual water snake Ss. A similar series of tests with individual garter snakes (Thamnophis radix) revealed significant preferences for areas soiled either by the $\mathrm{Ss}$ themselves or by conspecifics as compared to clean areas. No preferences were found for a clean area of the test chamber vs an area soiled by a sympatric water snake (Natrix r. r.). The possible role of chemical cues in the mediation of dispersion and social responsiveness was discussed.
\end{abstract}

Although reptilian species are typically believed to lead a relatively solitary existence (cf. Wynne-Edwards, 1962), several species of snakes are known to aggregate during certain times of the year for mating or hibernation, or in response to environmental factors such as changes in humidity or temperature (Noble \& Clausen, 1936). Since chemical stimuli are known to be of primary importance in mediating such overt behavior in snakes as feeding and responses to potential predators (Burghardt, 1970), social responsiveness (i.e., both aggregation and dispersal) may likewise be at least partially dependent upon chemical traces of conspecifics. Thus far, however, the behavioral responses of snakes to the chemical stimuli of conspecifics have been investigated in only a small number of species. Noble and Clausen (1936) have reported that aggregations in the common brown snakes (Storeria dekayi) appear to be mediated primarily through visual cues, with olfaction being of somewhat lesser importance. When working with ringneck snakes (Diadophis punctatus arnyi), however, Dundee and Miller (cited by Burghardt, 1970) found that their Ss preferred areas which had been previously soiled by conspecifics as compared to unsoiled areas. Likewise, blindsnakes (Leptotyphlops dulcis) are attracted to chemical secretions from conspecifics, while sympatric species are repelled by such chemical stimuli (Watkins, Gelbach, \& Kroll, 1969).

It should be emphasized that discrete species of snakes, like individual species of the more frequently studied aves and mammals, have evolved in their own unique fashions and may, therefore, display considerable interspecific variation in their modes of adapting to environmental contingencies. Therefore, generalizations to the entire order Squamata based upon the small

*This research was supported by NICHHD Grant 00973 . The authors wish to thank Gordon $M$. Burghardt, who supplied the Ss. number of representative species studied to date is certainly unwarranted.

In the study reported, approach and withdrawal behavior of water snakes (Natrix $r$. rhombifera) and garter snakes (Thamnophis radix) in response to chemical cues of conspecifics and of sympatric species of snakes is presented.

\section{EXPERIMENT I}

\section{Subjects}

Ten water snakes (Natrix r. rhombifera) laboratory born of a feral caught female were utilized in the present study. These snakes were 17 months of age at the start of the first experiment. Beginning at 2 months prior to the reported study, the 10 snakes were housed in two groups of five snakes each in glass aquaria.

\section{Apparatus}

Testing was conducted in 10 slate-bottom glass aquaria, each measuring $19 \times 10 \times 11.5$ in. high and covered with a porous metal lid. Each aquarium could be divided in to two equal sections $(9.5 \times 10 \times 11.5$ in. high) by the insertion of a tightly fitting plywood partition. Three rocks of approximately equal size were situated in each half of every aquarium.

\section{Procedure}

Chemical traces of snakes were deposited in one side only of each of the aquaria by confining a single snake in that half for $47.5 \mathrm{~h}$; the other half of each aquarium remained empty except for the three rocks. These stimulus snakes had access to a shallow bowl of water but were not fed during confinement (having been fed within $20 \mathrm{~h}$ prior to being placed in to the aquaria). All of the stimulus snakes defecated in their respective halves of the aquaria during the exposure period.

Immediately following the $47.5 \mathrm{~h}$ of confinement, the stimulus snake, water bowl, and plywood partition were removed from each of the aquaria, and one water snake was placed into the center of each enclosure (delineated by a strip of tape). The section of the aquarium (i.e., the side previously soiled by the stimulus snake vs the "clean" side) to which the $S$ initially moved was recorded. In a similar manner, the position of each snake was noted at 30-min intervals throughout an 8-h testing period. There were, thus, a total of 17 positional samples for each $S$.

Each water snake was tested twice, with an interval of 6 days 
Table 1

Responses of Water Snakes (Natrix r. rhombifera) to Soiled Vs Clean Halves of Test Chambers

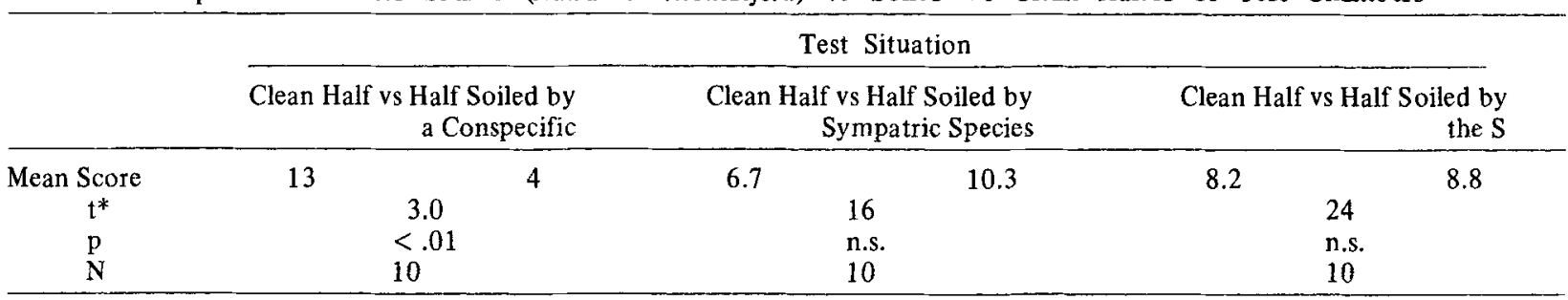

*Wilcoxon

between the two test sessions. In the first session, five Natrix $r . r$. were individually tested following exposure of half of the test aquarium to a conspecific (Natrix r. r.). The other five water snake Ss were tested for their positional preferences following exposure of one side of each aquarium to a garter snake (Thamnophis sirtalis sirtalis), a species whose geographical distribution overlaps that of the $\mathrm{S}$ species (Natrix r. r.) (Wright \& Wright, 1957). During the second test, the five water snakes that were previously tested in aquaria in half of which a conspecific had been previously confined were now tested in aquaria in which one side had housed a garter snake for the $47.5 \mathrm{~h}$ immediately preceding the test trial-and vice versa for the water snakes initially tested following garter snake presence in the test cage. In every instance, the conspecific chemical exposure water snake had not been housed together with the test $S$ for the 2 months preceding the test session (i.e., it had been housed in the other group cage). The interior of each aquarium was thoroughly cleaned between the two test sessions, and the Ss were rehoused in their original groups of five when not being tested.

\section{Results}

For statistical purposes, a preference score was computed for each S's response to each stimulus by subtracting the number of times that the $S$ was observed to be in the clean half of the aquarium from the number of times that it was on the soiled side (i.e., the side which had housed a stimulus snake). For example, a snake which was in the clean half of the aquarium during 12 of the sample observations and in the soiled half for the other five observations would have a preference score of -7 . Thus, a negative preference score indicates that the $S$ preferred the clean side of the aquarium over the soiled half during the 17 observations of the testing session, while a positive score indicates a preference for the soiled side.

The mean preference score for the water snakes tested in aquaria in which one-half had housed a conspecific prior to the testing (with the other half clean) was -9.0 (see Table 1). A Wilcoxon matched-pairs signed-ranks test indicates that the degree of preference for the clean side of the aquaria was significantly greater than for the side soiled by a conspecific water snake $(t=3.0, p<.01$, two-tailed test). When tested in aquaria in which one-half had previously held a garter snake (Thamnophis s. s.), however, no significant preferences were found (mean preference score $=+3.6, \mathrm{t}=16$ ) .

\section{EXPERIMENT II}

Experiment II was designed to ascertain whether or not the 10 water snakes would respond to their own chemical traces in the same manner as they had responded to conspecific chemical traces in Experiment $\mathrm{I}$.

\section{Procedure}

Three months after the completion of Experiment I; the same 10 water snakes were individually confined in one-half of an aquarium for $47.5 \mathrm{~h}$, with the other half remaining clean, as described previously. Immediately following this exposure period, each snake was removed briefly from its aquarium and replaced into the center following the removal of the plywood partition and water bowl. The Ss were then tested for their preferences for the side previously soiled by themselves vs the clean side of the aquaria, with the procedure being identical to that described for Experiment 1 .

\section{Results}

Five of the water snakes were most of ten observed in the halves of the aquaria which they had previously soiled, while the remaining five Ss were found most often in the clean halves of their respective aquaria (mean preference score $=0.6$ ). A Wilcoxon matched-pairs signed-ranks test indicated no significant preferences for either the clean side or the side which contained chemical traces of the Ss themselves $(t=24)$.

\section{EXPERIMENT III}

The cross-species generality of the results obtained in Experiment I were tested in a third series of experiments.

\section{Subjects}

Twenty-two wild-caught garter snakes (Thamnophis radix) were used as Ss in Experiment III. These snakes were fully adult at the time of testing and between 17.7 and $25.2 \mathrm{in.}$. in length.

\section{Apparatus}

Since the garter snakes used in Experiment III were considerably larger than the water snakes from the previous two experiments, larger testing chambers were required in this experiment. Testing chambers were constructed of galvanized steel and measured $48 \times 12 \times 12$ in. high. Each chamber was bottomless and was covered with a screen top. A tightly fitting insert was used to divide each chamber into two equal sections $(23.7 \times 12 \times 12$ in. high $)$.

\section{Procedure}

The procedure whereby chemical traces of either a conspecific or sympatric species of snake were deposited into one side of each test chamber (while the other half remained empty) was the 
Table 2

Responses of Garter Snakes (Thamnophis radix) to Soiled Vs Clean Halves of Test Chambers

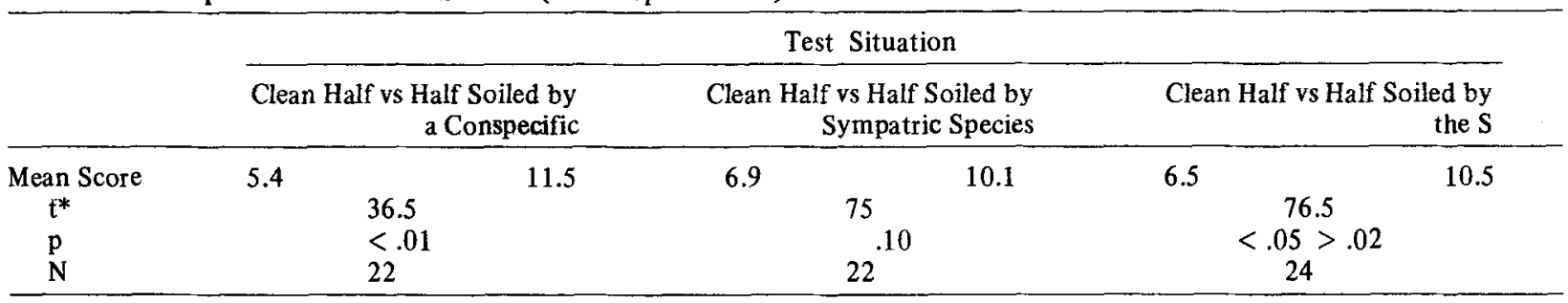

*Wilcoxon

same as for Experiment I. The sympatric species in this instance, however, was Natrix rhombifera. The bottomless test chambers were placed on to several folds of heavy brown paper (overlying a rubberized floor) which served as the substrate upon which the stimulus snakes were housed during the exposure period and the Ss subsequently tested.

Each $\mathrm{S}$ was tested once in each of the following two test situations: (1) one-half of the chamber soiled by a conspecific vs the other half clean and (2) one-half of the chamber soiled by a sympatric species (Natrix $r$.) vs the other half clean. The order of testing was counterbalanced across the $22 \mathrm{Ss}$ with an interval of 4 days between the two tests. Further details of testing and scoring are identical to those of Experiment $I$.

\section{Results}

The results of Experiment III are summarized in Table 2, with statistical treatment of the data being the same as for the two previous experiments. When given a choice between the clean half of the chamber vs the half previously soiled by a conspecific, the garter snakes showed a significant preference (mean preference score $=$ $6.1, \mathrm{p}<.01)$ for the conspecific soiled side. No significant preference was found for the side soiled by a sympatric water snake vs the clean side, however (mean preference score $=3.2$ ).

\section{EXPERIMENT IV}

In Experiment IV the 22 garter snakes used in Experiment III plus 2 additional snakes (total $=24$ ) were tested for their preferences for the half of the test chamber in which they had been individually housed for the preceding $47.5 \mathrm{~h}$ vs a clean half of that chamber. This experiment was, thus, a replication of Experiment II, but with garter snakes as Ss. Aside from the use of the larger test chambers (described for Experiment III), the methodological details are the same as for Experiment II. The interval between Experiments III and IV was approximately 2 months.

\section{Results}

The results from Experiment IV (summarized in the third column of Table 2) indicate that garter snakes preferred the side of the test chamber which they had soiled to the clean side (mean preference score $=4.0$, $\mathrm{p}<.05$ ).

\section{EXPERIMENT V}

While the experiments using water snakes were conducted in winter and early spring, the garter snake experiments took place in the autumn and in larger test chambers than used in the water snake experiments. Thus, the opposite responses of garter snakes and water snakes to conspecific chemical cues might have been a function of seasonal fluctuations in behavior or possibly related to the differing testing apparatus.

Experiment $\mathrm{V}$ is a partial replication of Experiment $\mathrm{I}$, with the original 10 water snakes now being tested in the autumn of the year and in the large chambers used for the garter snake tests.

\section{Procedure}

The 10 water snakes were tested in a single session each for their preferences for one-half of a test chamber which had been soiled by a conspecific vs a clean half of the chamber. The methodological details are the same as for Experiments I and III.

\section{Results}

The results for the 10 water snakes in Experiment $V$ indicate a significant preference for the clean side of the test chamber over the side soiled by a conspecific (mean preference score $=-6.2, p<.05$ ). These data, therefore, tend to corroborate those of Experiment I.

\section{DISCUSSION}

The results of the above experiments suggest that chemical traces of a conspecific may serve a communicative function in mediating spatial dispersion and/or social aggregations in garter snakes (Thamnophis radix) and water snakes (Natrix rhombifera). As suggested by Wynne-Edwards (1962) for carnivorous mammalian species, such long lasting chemical stimuli might function to "maintain the necessary social integration... even though any given individual may rarely come face to face with others, at least of his own sex."

While garter snakes showed a significant preference for an area soiled by a conspecific as compared to a clean area, water snakes tended to avoid an area previously soiled by a conspecific. However, both species displayed no consistent behavior in relation to areas soiled by sympatric species. The opposite behavior of the garter snakes and water snakes to conspecific chemical cues might be a reflection of the ecological differences between these two species. Alternatively, within each species similar chemical cues might possibly function to mediate both the dispersion and aggregation 
of individuals as a function of seasonal, climactic, or other variables (both internal and environmental).

The indiscriminate responsiveness of the water snakes to their own chemical traces in Experiment II, as compared to their avoidance of conspecific chemical cues in Experiment $I$, suggests that individuals are able to discriminate between their own chemical traces vs those of conspecifics. Such an ability to discriminate between the chemical traces of oneself as compared to those of conspecifics would, of course, be absolutely necessary for social avoidance responses mediated through chemical stimuli. That is, in order to effectively avoid conspecific chemical cues, the snake would have to be able to differentiate these cues from those constantly emanating from itself. Although the garter snakes did not respond differentially toward own vs conspecific soiled areas, this need not imply that they are unable to discriminate between these two sets of stimuli. Furthermore, it would seem that the ability to discriminate between own vs conspecific cues might be more critical where avoidance responses are involved than for positive responsiveness.

Finally, the nonresponsiveness of both species of snakes to the chemical cues of sympatric snakes suggests that responsiveness to the conspecific chemical cues appears to be a function of specific properties of these cues and not a response to chemical stimuli of sympatric species per se. Although the distributions of both water snakes and garter snakes overlap in large portions of the central United States (Wright \& Wright, 1957), they may not be serious competitors over prey species, and these two genera of snakes may, therefore, be of little direct importance to each other's survival. While garter snakes are found in all sorts of habitats throughout their home range, water snakes (as the name implies) are usually found in or around bodies or water (Wright \& Wright, 1957). Thus, water snakes typically feed upon such aquatic or semiaquatic prey as fish, toads, frogs, and crayfish (Wright \& Wright, 1957), while garter snakes appear to have a more eclectic diet, including amphibians, small mammals, insects, fish, young birds, and earthworms (Wright \& Wright, 1957; Burghardt, 1970).

\section{REFERENCES}

Burghardt, G. M. Chemical perception in reptiles. In J. W. Johnston, Jr., D. G. Moulton, and A. Turk (Eds.), Communication by chem
Appleton-Century-Crofts, 1970 .

Noble, G. K., \& Clausen, H. J. The aggregation behavior of Storeria dekay $i$ and other snakes, with especial reference to the sense organs involved. Ecological Monographs, 1936, 6, 270-316.

Watkins, J. F., Gehlbach, F. R., \& Kroll, J. C. Attractant-repellent secretions of blind snakes (Leptotyphlops dulcis) and their army ant prey (Neivamyrmex nigrescens). Ecology, 1969, 50, 1098-1102.

Wright, A. H., \& Wright, A. A. Handbook of snakes. Ithaca, N.Y:

Comstock, 1957 .
Wynne-Edwards, V. C. Animal dispersion in relation to social behaviour, New York: Hafner, 1962.

(Received for publication May 7, 1973; revision received January $9,1974$. ) 